\title{
Relationships between Alanine Aminotransferase (ALT), Visceral Adipose Tissue (AT) and Metabolic Risk Factors in a Middle-Aged Japanese Population
}

\author{
Rie Oka ${ }^{1}$, Kunimasa Yagi², Chiaki Nakanishi², Tetsuo Konno², Masa-aki Kawashiri², Kenshi Hayashi², \\ Atsushi Nohara ${ }^{3}$, Akihiro Inazu ${ }^{4}$ and Masakazu Yamagishi ${ }^{2}$ \\ ${ }^{1}$ Department of Internal Medicine, Hokuriku Central Hospital, Toyama, Japan \\ ${ }^{2}$ Department of Internal Medicine, Kanazawa University Graduate School of Medical Science, Kanazawa, Japan \\ ${ }^{3}$ Department of Lipidology, Kanazawa University Graduate School of Medical Science, Kanazawa, Japan \\ ${ }^{4}$ Department of Laboratory Science, School of Health Sciences, Kanazawa University Graduate School of Medical Science, Kanazawa, \\ Japan
}

\begin{abstract}
Aim: The commonly observed relationship between increased visceral adiposity and metabolic abnormalities may be partly mediated by a concomitant increase in liver fat content. We evaluated the independent association between the level of alanine aminotransferase (ALT) as a surrogate marker of the liver fat content and the incidence of metabolic abnormalities after adjusting for the amount of visceral adipose tissue (AT).

Methods: The subjects included 1,118 Japanese individuals (44\% women) who underwent computed tomography to assess the amount of visceral AT on medical checkups. Cross-sectional associations between the serum ALT, visceral AT and metabolic risk factors were examined.

Results: The ALT level and visceral AT were found to show a significant correlation $(r=0.41$ in men and $r=0.36$ in women, $p<0.001)$. In a multivariable linear regression analysis, the ALT level and visceral AT were found to be independently associated with blood pressure in men and triglycerides and 2-hour post-challenge glucose in both genders $(p<0.01)$, whereas only visceral AT was found to be associated with HDL-cholesterol $(p<0.01)$. When the participants were classified into four subgroups based on the 75th percentiles of ALT and visceral AT, the low-ALT/high-visceral AT group, but not the high-ALT/low-visceral AT group, had a significantly higher odds ratio for low HDL-cholesterol among both genders $(p<0.05)$ and for hypertriglyceridemia in men only $(p<0.05)$. Meanwhile, the high-ALT/low-visceral AT group, but not the low-ALT/high-visceral AT group, had a significantly higher odds ratio for IGT among women $(p<0.05)$.

Conclusions: Although the ALT level and visceral AT were found to be independently associated with most metabolic risk factors, visceral AT had a dominant association with dyslipidemia in both genders, while the ALT level appeared to have a closer association with IGT in women.
\end{abstract}

J Atheroscler Thromb, 2014; 21:582-592.

Key words: Obesity, Fatty liver, Visceral adiposity, IGT

\section{Introduction}

Although the close link between increased vis-

Address for correspondence: Rie Oka, Department of Internal Medicine, Hokuriku Central Hospital, 123 Nodera, Oyabe, Toyama, 932-8503, Japan

E-mail: ririoka@goo.jp

Received: October 9, 2013

Accepted for publication: December 20, 2013 ceral adiposity and metabolic abnormalities has been extensively studied ${ }^{1,2)}$, it may be partly mediated by a concomitant increase in the liver fat content ${ }^{3,4)}$. A high liver fat content can by itself explain the dysregulation of lipid and carbohydrate metabolism without involving visceral $\mathrm{AT}^{5)}$. Some investigators have reported that the liver fat content has an even stronger association with insulin resistance ${ }^{6,7)}$ and very-lowdensity lipoprotein-triglyceride (VLDL-TG) produc- 
tion $^{8)}$ than visceral AT.

The alanine aminotransferase (ALT) level is considered to reflect the liver fat content when other causes of fatty liver, such as alcohol consumption and viral hepatitis, are excluded, even among non-obese individuals within the reference range for $\mathrm{ALT}^{9}$ ). The ALT level has been shown to be well correlated with the amount of directly measured liver fat on magnetic resonance spectroscopy $(r=0.46$ in women and 0.62 in men ${ }^{10)}$. Because measuring the serum ALT level is relatively inexpensive and easy compared to performing radiological imaging techniques or liver biopsies, this parameter has been used as a surrogate marker of the liver fat content in epidemiological studies. Several cross-sectional and prospective studies have demonstrated that the ALT levels are independently associated with incident diabetes and the constellation of metabolic risk factors after adjusting for parameters of adiposity, such as body mass index (BMI) and waist circumference ${ }^{11-13)}$. Recently, the Framingham Heart Study demonstrated an independent association between the ALT level and various metabolic risk factors, even after adjusting for visceral $\mathrm{AT}^{14)}$. Because the Japanese population has a higher propensity toward liver fat accumulation at lower BMI values ${ }^{15)}$, the impact of ALT on metabolic deterioration may be stronger than that observed in Caucasians.

In this study, we performed a cross-sectional analysis of the relationships between the ALT level, amount of visceral AT and presence of metabolic risk factors in apparently healthy Japanese adults. Specifically, we sought to compare the relative impact of the ALT level and visceral AT on each risk factor among middle-aged, non-diabetic Japanese men and women.

\section{Subjects and Methods}

\section{Study Sample}

The study protocol was approved by the hospital review board of Hokuriku Central Hospital. This hospital has a special department where public school employees can receive routine medical checkups, which are mandated by law and sponsored by their mutual aid association. Among the employees who received regular checkups between April 2006 and March 2010, 1,160 individuals voluntarily underwent computed tomography (CT) to evaluate their abdominal fat distribution as well as OGTT testing. All subjects were Japanese men and women 30-62 years of age; $98 \%$ were teachers. After excluding subjects with a fasting plasma glucose level of $\geq 126 \mathrm{mg} / \mathrm{dL} \quad(n=26)$, those taking steroids $(n=4)$ or hormone replacement therapy $(n=3)$ and those positive for hepatitis B virus surface antigens $(n=7)$ or hepatitis $C$ virus antibodies $(n=3)$, the remaining 1,118 individuals $(630$ men and 488 women) were ultimately enrolled in this study. The participants were considered smokers if they smoked at least one cigarette per day. Alcohol use was defined according to the number of days per week that an alcoholic drink was consumed, regardless of the amount. Women reporting no menses for at least six months were considered to be menopausal, and those with a history of hysterectomy were considered to be postmenopausal if they were over the age of 51 years, which was the average menopausal age of this sample. Signed informed consent was obtained from all participants.

\section{Blood Sampling and Anthropometric Measurements}

All participants were asked to visit our hospital between 8:00 a.m. and 9:00 a.m. after an overnight fast. Blood samples were drawn from the antecubital vein to measure the levels of ALT, aspartate aminotransferase (AST), triglycerides and high-density lipoprotein (HDL)-cholesterol. OGTT testing (75 g of dextrose monohydrate in $250 \mathrm{~mL}$ water) was subsequently performed to establish the fasting and 2-hour plasma glucose levels. The levels of ALT, AST, triglycerides and HDL-cholesterol were measured using enzymatic analytical chemistry (Autoanalyzer BioMajesty JCA-BM1650, JEOL Ltd., Tokyo, Japan), while the plasma glucose levels were assessed using the glucose oxidase method (Automatic Glucose Analyzer ADAMS Glucose GA-1160, Arkray, Kyoto) in the hospital laboratory.

The anthropometric measurements were obtained according to previously published methods ${ }^{16)}$. Blood pressure was measured twice in the sitting position using an automatic device (Colin Model BP-203RV, Colin, Tokyo, Japan) after at least five minutes of rest. The average of the two readings was used as the blood pressure value.

\section{Assessment of Metabolic Risk Factors}

Metabolic risk factors were defined according to the National Cholesterol Education Program (NCEP) Adult Treatment Panel III definition of metabolic syndrome $^{17)}$ : 1) high blood pressure, $\geq 130 / 85 \mathrm{mmHg}$ or the use of antihypertensive medications; 2) hypertriglyceridemia, $\geq 150 \mathrm{mg} / \mathrm{dL}(1.69 \mathrm{mmol} / \mathrm{L}) ; 3)$ low HDL-cholesterol, $<40 \mathrm{mg} / \mathrm{dL}(1.04 \mathrm{mmol} / \mathrm{L})$ for men and $<50 \mathrm{mg} / \mathrm{dL}(1.04 \mathrm{mmol} / \mathrm{L})$ for women; 4$)$ impaired fasting plasma glucose (IFG), $\geq 100 \mathrm{mg} / \mathrm{dL}$ (5.6 $\mathrm{mmol} / \mathrm{L})$; and 5) impaired glucose tolerance (IGT), 2-hour post-challenge plasma glucose $\geq 140$ $\mathrm{mg} / \mathrm{dL}(7.0 \mathrm{mmol} / \mathrm{L})$. 
Measurement of Abdominal Adipose Tissue on CT

Detailed methods for the measurement of abdominal adipose tissue on CT have been previously published ${ }^{16)}$. Briefly, an axial CT scan was obtained at the level of the umbilicus in each participant using an electron beam CT scanner (Aquilion Toshiba Medical Systems, Tokyo, Japan). Planimetric measurements obtained at the level of the umbilicus have been reported to be well correlated with the values of volumetric quantification of visceral AT $(r=0.81$ in men and $r=0.85$ in women, $p<0.001)$ and SAT $(r=0.95$ in men and $r=0.92$ in women, $p<0.001)^{18)}$. The images generated were transferred to a work station and analyzed using a commercial software program designed to quantify the amount of visceral AT (Fat Scan version 3.0, N2 System, Osaka, Japan). The interobserver intraclass correlation coefficient between two observers analyzing the same images of visceral AT $(n=30)$ was 0.98 .

\section{Statistical Analysis}

All statistical analyses were performed separately for men and women. Simple correlations between the ALT level, visceral AT and metabolic risk factors were assessed using Spearman correlation coefficients. The ALT values were logarithmically transformed before the analysis due to their skewed distribution. A multivariable linear regression analysis was used to assess the associations between the log ALT values and the continuous metabolic risk factors. The log ALT, visceral AT and BMI values were first standardized to a mean of 0 and standard deviation (SD) of 1 within each sex and then included in the regression models, and the average change in each risk factor per a 1-SD increase in each variable was estimated. First, only log ALT, visceral AT or BMI were solely included in the model. Subsequently, log ALT and visceral AT (Model 1) or log ALT and BMI (Model 2) were jointly included to assess whether the log ALT values were associated with metabolic risk factors independently of visceral AT (Model 1) or BMI (Model 2). A logistic regression analysis was used to assess the independent associations between the log ALT and visceral AT values (Model 1) or the log ALT and BMI values (Model 2) with respect to the presence of metabolic risk factors. Finally, the participants were classified into four subgroups according to their ALT and visceral AT values using the 75th percentile as the cutoff point: lowALT/low-visceral AT, high-ALT/low-visceral AT, lowALT/high-visceral AT and high-ALT/high-visceral AT. The adjusted odds ratio of having a metabolic risk factor was calculated in each subgroup relative to a reference group (low-ALT/low-visceral AT). All regression
Table 1. Clinical characteristics of the study participants

\begin{tabular}{lcc}
\hline \multicolumn{1}{c}{ Characteristics } & $\begin{array}{c}\text { Men } \\
(n=630)\end{array}$ & $\begin{array}{c}\text { Women } \\
(n=488)\end{array}$ \\
\hline Anthropometry and adiposity parameters & \\
Age (years) & $51.6 \pm 7.1$ & $53.3 \pm 6.5^{*}$ \\
Height $(\mathrm{cm})$ & $170.0 \pm 5.8$ & $156.6 \pm 5.5^{*}$ \\
Weight $(\mathrm{kg})$ & $73.3 \pm 10.2$ & $58.5 \pm 8.0^{*}$ \\
Body mass index $\left(\mathrm{kg} / \mathrm{m}^{2}\right)$ & $25.3 \pm 2.9$ & $23.8 \pm 3.1^{*}$ \\
Waist circumference $(\mathrm{cm})$ & $88.0 \pm 7.5$ & $83.0 \pm 8.2^{*}$ \\
Visceral AT (cm $\left.{ }^{2}\right)$ & $141.2 \pm 50.5$ & $83.0 \pm 38.3^{*}$ \\
Metabolic parameters & & \\
Systolic BP (mmHg) & $130.6 \pm 15.3$ & $127.5 \pm 19.6^{*}$ \\
Diastolic BP (mmHg) & $81.5 \pm 9.5$ & $77.6 \pm 11.5^{*}$ \\
Fasting PG (mg/dL) & $99.3 \pm 9.2$ & $95.4 \pm 8.7^{*}$ \\
2-hour PG (mg/dL) & $123.7 \pm 32.5$ & $119.0 \pm 28.4^{*}$ \\
Triglycerides (mg/dL) & $92 / 123 / 174$ & $66 / 91 / 127^{*}$ \\
HDL-cholesterol (mg/dL) & $54.3 \pm 12.0$ & $63.7 \pm 14.2^{*}$ \\
ALT (U/L) & $20 / 26 / 38$ & $14 / 18 / 23^{*}$ \\
Medications and Habitts & & \\
Antgi-hypertensive medication & 16.3 & $12.9^{*}$ \\
Lipids-lowering medication & 9.2 & 10.0 \\
Current cigarette smoker $(\%)$ & 22.4 & $1.4^{*}$ \\
Drinking everyday & 33.7 & $7.4^{*}$ \\
Drinking 1-6 days per week & 40.0 & $28.9^{*}$ \\
Postmenopausal & & 64.5 \\
\hline
\end{tabular}

Data are mean \pm SD, 25/50/75th percentile values, or $\%$. AT, adipose tissue; BP,blood pressure; PG, plasma glucose; HDL, high density lipid; ALT, alanineaminotransferase. ${ }^{*} p<0.05$ between men and women.

models included the following variables as covariates: age, smoking, alcohol use (three-level variables: drinking everyday/drinking one to six days per week/drinking less than one day per week), use of medications for hypertension or dyslipidemia and the menopause status (women only). The analyses of the triglyceride levels were conducted after logarithmic transformation. A $p$ value of $<0.05$ was considered to be statistically significant, and all analyses were performed using the SPSS software package version 11.0 for Windows (SPSS Inc. Chicago, USA).

\section{Results}

The clinical characteristics of the participants are presented in Table 1. The mean age of the study sample was slightly over 50 years for both genders. The mean BMI was $25.3 \mathrm{~kg} / \mathrm{m}^{2}$ for men and $23.8 \mathrm{~kg} / \mathrm{m}^{2}$ for women. The median ALT was $26 \mathrm{U} / \mathrm{L}$ in men and $18 \mathrm{U} / \mathrm{L}$ in women. The mean visceral AT was approximately 1.5 -fold larger in men than in women. Men 
Table 2. Correlation coefficients of ALT, visceral AT, and cardiometabolic risk factors

\begin{tabular}{lccccc}
\hline & \multicolumn{2}{c}{ Men $(n=630)$} & & \multicolumn{2}{c}{ Women $(n=488)$} \\
\cline { 2 - 3 } \cline { 5 - 6 } & ALT & Visceral AT & & ALT & Visceral AT \\
\hline Visceral AT & $0.41^{* *}$ & 1.00 & & $0.36^{* *}$ & 1.00 \\
Subcutaneous AT & $0.30^{* *}$ & $0.50^{* *}$ & & $0.21^{* *}$ & $0.57^{* *}$ \\
Body mass index & $0.43^{* *}$ & $0.59^{* *}$ & & $0.27^{* *}$ & $0.66^{* *}$ \\
Age & $-0.13^{*}$ & $0.11^{*}$ & & $0.22^{* *}$ & $0.21^{* *}$ \\
Systolic BP & $0.23^{* *}$ & $0.31^{* *}$ & & $0.17^{* *}$ & $0.31^{* *}$ \\
Dyastolic BP & $0.21^{* *}$ & $0.30^{* *}$ & & $0.17^{* *}$ & $0.29^{* *}$ \\
Triglycirides & $0.29^{* *}$ & $0.33^{* *}$ & & $0.35^{* *}$ & $0.43^{* *}$ \\
HDL cholesterol & $-0.20^{* *}$ & $-0.22^{* *}$ & & $-0.17^{* *}$ & $-0.40^{* *}$ \\
Fasting PG & $0.15^{* *}$ & $0.15^{* *}$ & & $0.24^{* *}$ & $0.28^{* *}$ \\
2-hour PG & $0.24^{* *}$ & $0.27^{* *}$ & & $0.26^{* *}$ & $0.28^{* *}$ \\
\hline
\end{tabular}

Abbreviations are same as Table $1 .{ }^{* *} p<0.001 ;{ }^{*} p<0.01$

showed more adverse levels of the most continuous risk factors than women. Although diabetic participants were excluded as described above, some subjects were taking medications for hypertension and/or dyslipidemia.

Table 2 shows the Spearman correlation coefficients for ALT and visceral AT with the metabolic risk factors. The ALT level and visceral AT were positively correlated in both genders $(r=0.41$ in men and $r=$ 0.36 in women, $p<0.001)$. The correlation between the ALT level and age was positive in women $(r=0.22$, $p<0.001)$ and inverse in men $(r=-0.13, p<0.01)$. Both the ALT level and visceral AT were significantly correlated with all metabolic risk factors in both genders $(p<0.001)$. The correlation coefficients for AST and metabolic risk factors were consistently weaker than those for ALT, and further multivariate analyses were not performed.

The results of the multiple regression analysis are shown in Table 3. After adjusting for covariates such as age, smoking, alcohol use, use of medications and the menopausal status (women only), the log ALT, visceral AT and BMI values were each found to be positively associated with blood pressure, plasma glucose and $\log$ triglyceride values and inversely associated with the HDL-cholesterol levels $(p<0.01)$. For example, as shown in the furthest left column, the systolic blood pressure values increased $3.76 \pm 0.58 \mathrm{mmHg}$, $4.25 \pm 0.59 \mathrm{mmHg}$ and $6.21 \pm 0.55 \mathrm{mmHg}$ per each $1-S D$ increase in the log ALT, visceral AT and BMI values, respectively, after adjusting for the covariates in men. When log ALT was included jointly with visceral AT (Model 1) or BMI (Model 2) as independent variables, it was found to be independently associated with blood pressure in men $(p<0.01)$ but not in women $(p>0.05)$. The association with $\log$ ALT was independent of visceral AT (Model 1) and BMI (Model 2) for triglycerides and plasma glucose in both sexes $(p<0.05)$, except for fasting plasma glucose in men. Regarding HDL-cholesterol, the association with $\log$ ALT was diminished after adjusting for visceral AT (Model 1), but not BMI, in both sexes (Model 2) $(p<0.05)$. When subcutaneous AT and log ALT were jointly included as independent variables, subcutaneous AT was also found to be independently associated with most metabolic risk factors, although the associations were not as strong as those for visceral AT to diminish the association between log ALT and HDL-cholesterol in both sexes and 2-hour plasma glucose in women (data not shown). Finally, when the analysis was confined to non-drinkers, the association with $\log$ ALT remained independent of visceral AT for blood pressure and plasma glucose in men $(p<0.05)$ and plasma glucose and triglycerides in women $(p<$ 0.05) (Supplementary Table).

Table 4 shows the results of the logistic regression analysis of dichotomous risk factors. In Model 1 , both $\log$ ALT and visceral AT were found to be independently associated with most of the metabolic risk factors tested $(p<0.05)$, except for low HDL-cholesterol in both genders and hypertension in women. In Model 2, only log ALT was found to be independently associated with IGT in both sexes and hypertriglyceridemia in women $(p<0.05)$.

Table 5 shows the adjusted odds ratios for risk factors among the four subgroups based on the ALT and visceral AT levels. Compared to the reference group (low-ALT/low-visceral AT), the low-ALT/highvisceral AT group, but not the high-ALT/low-visceral AT group, had a significantly higher odds ratio for low 
Table 3. Multivariable-adjusted* regressions for continuous metabolic risk factors with Log ALT, Visceral AT, and BMI

\begin{tabular}{|c|c|c|c|c|c|c|c|c|c|}
\hline & \multicolumn{3}{|c|}{$\begin{array}{c}\text { Log ALT, Visceral AT, or BMI } \\
\text { solely included }\end{array}$} & \multicolumn{3}{|c|}{$\begin{array}{l}\text { Log ALT and Visceral AT } \\
\text { jointly included (Model 1) }\end{array}$} & \multicolumn{3}{|c|}{$\begin{array}{c}\text { Log ALT and BMI } \\
\text { jointly included (Model 2) }\end{array}$} \\
\hline & $\beta$ & t-statistic & $p$ value & $\beta$ & t-statistic & $p$ value & $\beta$ & t-statistic & $p$ value \\
\hline \multicolumn{10}{|l|}{$\operatorname{Men}(n=630)$} \\
\hline \multicolumn{10}{|c|}{ Systolic blood pressure (mmHg) } \\
\hline Log ALT & $3.76 \pm 0.58$ & 6.46 & $<0.01$ & $2.50 \pm 0.62$ & 4.00 & $<0.01$ & $1.48 \pm 0.60$ & 2.47 & 0.01 \\
\hline Visceral AT & $4.25 \pm 0.59$ & 7.23 & $<0.01$ & $3.24 \pm 0.63$ & 5.12 & $<0.01$ & & & \\
\hline BMI & $6.21 \pm 0.55$ & 11.29 & $<0.01$ & & & & $5.60 \pm 0.60$ & 9.34 & $<0.01$ \\
\hline \multicolumn{10}{|c|}{ Diastolic blood pressure (mmHg) } \\
\hline Log ALT & $2.24 \pm 0.36$ & 6.18 & $<0.01$ & $1.50 \pm 0.39$ & 3.86 & $<0.01$ & $0.94 \pm 0.38$ & 2.49 & 0.01 \\
\hline Visceral AT & $2.49 \pm 0.37$ & 6.81 & $<0.01$ & $1.88 \pm 0.39$ & 4.78 & $<0.01$ & & & \\
\hline BMI & $3.57 \pm 0.35$ & 10.34 & $<0.01$ & & & & $3.18 \pm 0.38$ & 8.46 & $<0.01$ \\
\hline \multicolumn{10}{|c|}{ Fasting plasma glucose $(\mathrm{mg} / \mathrm{dL})$} \\
\hline Log ALT & $1.29 \pm 0.37$ & 3.49 & $<0.01$ & $0.99 \pm 0.40$ & 2.47 & 0.01 & $0.97 \pm 0.40$ & 2.40 & 0.02 \\
\hline Visceral AT & $1.16 \pm 0.38$ & 3.09 & $<0.01$ & $0.76 \pm 0.41$ & 1.86 & 0.06 & & & \\
\hline BMI & $1.18 \pm 0.37$ & 3.19 & $<0.01$ & & & & $0.79 \pm 0.40$ & 1.95 & 0.05 \\
\hline \multicolumn{10}{|c|}{ 2-hour plasma glucose (mg/dL) } \\
\hline Log ALT & $8.17 \pm 1.26$ & 6.51 & $<0.01$ & $5.87 \pm 1.35$ & 4.35 & $<0.01$ & $6.93 \pm 1.37$ & 5.05 & $<0.01$ \\
\hline Visceral AT & $8.27 \pm 1.28$ & 6.47 & $<0.01$ & $5.89 \pm 1.37$ & 4.29 & $<0.01$ & & & \\
\hline BMI & $5.88 \pm 1.28$ & 4.60 & $<0.01$ & & & & $3.05 \pm 1.37$ & 2.22 & 0.03 \\
\hline \multicolumn{10}{|c|}{ Log triglycerides $(\mathrm{mg} / \mathrm{dL})$} \\
\hline Log ALT & $0.12 \pm 0.02$ & 6.37 & $<0.01$ & $0.06 \pm 0.02$ & 3.36 & $<0.01$ & $0.09 \pm 0.02$ & 4.42 & $<0.01$ \\
\hline Visceral AT & $0.17 \pm 0.02$ & 8.77 & $<0.01$ & $0.14 \pm 0.02$ & 6.78 & $<0.01$ & & & \\
\hline BMI & $0.11 \pm 0.02$ & 5.78 & $<0.01$ & & & & $0.07 \pm 0.02$ & 3.55 & $<0.01$ \\
\hline \multicolumn{10}{|c|}{ HDL-cholesterol (mg/dL) } \\
\hline Log ALT & $-1.90 \pm 0.47$ & -4.01 & $<0.01$ & $-0.90 \pm 0.50$ & -1.80 & 0.07 & $-1.22 \pm 0.51$ & -2.40 & 0.02 \\
\hline Visceral AT & $-2.92 \pm 0.47$ & -6.24 & $<0.01$ & $-2.56 \pm 0.51$ & -5.02 & $<0.01$ & & & \\
\hline BMI & $-2.17 \pm 0.47$ & -4.64 & $<0.01$ & & & & $-1.67 \pm 0.51$ & -3.27 & $<0.01$ \\
\hline
\end{tabular}

HDL-cholesterol in both genders $(p<0.05$ in men and $p<0.001$ in women) and hypertriglyceridemia in men $(p<0.05)$. The high-ALT/low-visceral AT group, but not the low-ALT/high-visceral AT group, had a significantly higher odds ratio for IGT in women $(p<$ 0.05). The high-ALT/high-visceral AT group had the highest odds ratios for all risk factors, except low HDL-cholesterol.

\section{Discussion}

In the present study, we performed a cross-sectional analysis of the associations between the ALT level, visceral AT and metabolic risk factors in middleaged, non-diabetic Japanese men and women. The primary finding of this study is that the ALT level exhibits a significant association with most metabolic risk factors, except HDL-cholesterol, even after adjust- ing for visceral AT. This result suggests that the ALT level is a significant clinical indicator of pathological fat distribution, independent of visceral AT, in the Japanese population. Categorization into four subgroups based on the ALT and visceral AT values revealed that women with an elevated ALT level, but not increased amount of visceral AT, had a higher odds ratio for IGT, indicating that the impact of ALT on post-challenge plasma glucose may be even stronger than that of visceral AT.

The ALT and visceral AT values were found to be interrelated, in agreement with the findings of prior studies $6,7,14,19)$. The correlation coefficients observed in the current study $(r=0.41$ in men and $r=0.36$ in women) are comparable to those reported in the Framingham Heart Study (0.31 in men and 0.21 in women $)^{14)}$ and the Jackson Heart Study $(-0.30)^{19)}$, although in the latter study, the liver fat content was 
(Cont Table 3)

\begin{tabular}{|c|c|c|c|c|c|c|c|c|c|}
\hline & \multicolumn{3}{|c|}{$\begin{array}{l}\text { Log ALT, Visceral AT, or BMI } \\
\text { solely included }\end{array}$} & \multicolumn{3}{|c|}{$\begin{array}{l}\text { Log ALT and Visceral AT } \\
\text { jointly included (Model 1) }\end{array}$} & \multicolumn{3}{|c|}{$\begin{array}{c}\text { Log ALT and BMI } \\
\text { jointly included (Model 2) }\end{array}$} \\
\hline & $\beta$ & t-statistic & $p$ value & $\beta$ & t-statistic & $p$ value & $\beta$ & t-statistic & $p$ value \\
\hline \multicolumn{10}{|c|}{ Women $(n=488)$} \\
\hline \multicolumn{10}{|c|}{ Systolic blood pressure (mmHg) } \\
\hline Log ALT & $3.21 \pm 0.88$ & 3.66 & $<0.01$ & $1.48 \pm 0.91$ & 1.62 & 0.11 & $1.47 \pm 0.90$ & 1.63 & 0.10 \\
\hline Visceral AT & $5.41 \pm 0.86$ & 6.32 & $<0.01$ & $4.89 \pm 0.92$ & 5.34 & $<0.01$ & & & \\
\hline BMI & $5.56 \pm 0.83$ & 6.71 & $<0.01$ & & & & $5.09 \pm 0.88$ & 5.79 & $<0.01$ \\
\hline \multicolumn{10}{|c|}{ Diastolic blood pressure $(\mathrm{mmHg})$} \\
\hline Log ALT & $1.74 \pm 0.52$ & 3.37 & $<0.01$ & $0.79 \pm 0.54$ & 1.47 & 0.14 & $0.70 \pm 0.53$ & 1.32 & 0.19 \\
\hline Visceral AT & $2.95 \pm 0.51$ & 5.83 & $<0.01$ & $2.67 \pm 0.54$ & 4.94 & $<0.01$ & & & \\
\hline BMI & $3.26 \pm 0.49$ & 6.70 & $<0.01$ & & & & $3.04 \pm 0.52$ & 5.88 & $<0.01$ \\
\hline \multicolumn{10}{|c|}{ Fasting plasma glucose $(\mathrm{mg} / \mathrm{dL})$} \\
\hline Log ALT & $1.85 \pm 0.38$ & 4.88 & $<0.01$ & $1.25 \pm 0.40$ & 3.14 & $<0.01$ & $1.28 \pm 0.40$ & 3.24 & $<0.01$ \\
\hline Visceral AT & $2.13 \pm 0.38$ & 5.66 & $<0.01$ & $1.69 \pm 0.40$ & 4.23 & $<0.01$ & & & \\
\hline BMI & $2.08 \pm 0.37$ & 5.67 & $<0.01$ & & & & $1.66 \pm 0.39$ & 4.32 & $<0.01$ \\
\hline \multicolumn{10}{|c|}{ 2-hour plasma glucose (mg/dL) } \\
\hline Log ALT & $7.50 \pm 1.26$ & 5.97 & $<0.01$ & $5.94 \pm 1.33$ & 4.47 & $<0.01$ & $6.24 \pm 1.32$ & 4.72 & $<0.01$ \\
\hline Visceral AT & $6.52 \pm 1.27$ & 5.13 & $<0.01$ & $4.41 \pm 1.33$ & 3.31 & $<0.01$ & & & \\
\hline BMI & $5.70 \pm 1.24$ & 4.59 & $<0.01$ & & & & $3.67 \pm 1.29$ & 2.84 & $<0.01$ \\
\hline \multicolumn{10}{|c|}{ Log triglycerides (mg/dL) } \\
\hline Log ALT & $0.16 \pm 0.02$ & 7.63 & $<0.01$ & $0.10 \pm 0.02$ & 4.92 & $<0.01$ & $0.14 \pm 0.02$ & 6.26 & $<0.01$ \\
\hline Visceral AT & $0.19 \pm 0.02$ & 9.47 & $<0.01$ & $0.16 \pm 0.02$ & 7.32 & $<0.01$ & & & \\
\hline BMI & $0.11 \pm 0.02$ & 5.19 & $<0.01$ & & & & $0.06 \pm 0.02$ & 3.00 & $<0.01$ \\
\hline \multicolumn{10}{|c|}{ HDL-cholesterol (mg/dL) } \\
\hline Log ALT & $-2.65 \pm 0.65$ & -4.09 & $<0.01$ & $-0.75 \pm 0.65$ & -1.16 & 0.25 & $-0.15 \pm 0.67$ & -2.20 & 0.03 \\
\hline Visceral AT & $-5.63 \pm 0.61$ & -9.25 & $<0.01$ & $-5.36 \pm 0.65$ & -8.24 & $<0.01$ & & & \\
\hline BMI & $-3.92 \pm 0.62$ & -6.35 & $<0.01$ & & & & $-3.44 \pm 0.65$ & -5.28 & $<0.01$ \\
\hline
\end{tabular}

*All regression models are adjusted for age, alcohole intake, smoking, taking medications, and menopause (women only). $\beta$ denotes the average change $\left( \pm\right.$ SE) in risk factor per 1-SD increase in Log ALT, Visceral AT, or BMI. The values of 1-SD are 0.51 and 0.45 for Log ALT, $50 \mathrm{~cm}^{2}$ and 38 $\mathrm{cm}^{2}$ for Visceral AT, and $2.9 \mathrm{~kg} / \mathrm{m}^{2}$ and $3.1 \mathrm{~kg} / \mathrm{m}^{2}$ for BMI in men and women, respectively.

assessed according to the degree of liver attenuation on CT. However, using the top quartile level as a cutoff point in the group comparisons in this study, the overlap between a high ALT level and a high visceral AT value was at most half. In Asians, nonalcoholic fatty liver disease (NAFLD) is not rare among nonobese adults ${ }^{20)}$. Even when evaluated according to visceral AT, the presence of obesity and an elevated ALT level can be dissociated.

The ALT and visceral AT values were found to be independently associated with most metabolic risk factors in both men and women. A recent report from the Framingham Heart Study also demonstrated an independent association between the ALT level and metabolic risk factors after adjusting for visceral AT and insulin resistance ${ }^{14)}$. Similar results have also been documented in the Jackson Heart Study, which was conducted among black men ${ }^{19)}$. In contrast, in small studies of obese subjects, Fabrrini et al. found that increased visceral AT values are not associated with increased insulin resistance or VLDL-TG production rates unless accompanied by increases in the liver fat content ${ }^{4,21)}$. These discrepancies may be due to the smaller range of BMI and visceral AT values in that study. In general populations not selected for obesity, adjusting for visceral AT is not likely to eliminate the association with ALT for most metabolic complications.

The presence of visceral AT abolished the association between the ALT and HDL-cholesterol level in both men and women, consistent with the results of the abovementioned cohort studies ${ }^{14,19)}$ and a recently 
Table 4. Logistic regressions for metabolic risk factors with 1-SD increase in log ALT, Visceral AT, or BMI

\begin{tabular}{|c|c|c|c|c|c|c|c|c|}
\hline & \multicolumn{4}{|c|}{ Men $(n=630)$} & \multicolumn{4}{|c|}{ Women $(n=488)$} \\
\hline & Odds ratio & $p$ value & Odds ratio & $p$ value & Odds ratio & $p$ value & Odds ratio & $p$ value \\
\hline \multicolumn{9}{|l|}{ Hypertension } \\
\hline Log ALT & $1.35(1.11-1.64)$ & $<0.01$ & $1.25(1.03-1.53)$ & 0.03 & $1.14(0.92-1.41)$ & 0.22 & $1.15(0.93-1.42)$ & 0.19 \\
\hline BMI & & & $2.03(1.62-2.54)$ & $<0.01$ & & & $1.69(1.36-2.10)$ & $<0.01$ \\
\hline \multicolumn{9}{|c|}{ Impaired fasting glucose (IFG) } \\
\hline Log ALT & $1.20(1.00-1.44)$ & 0.05 & $1.20(1.00-1.44)$ & 0.05 & $1.26(1.01-1.57)$ & 0.04 & $1.27(1.01-1.58)$ & 0.04 \\
\hline Visceral AT & $1.30(1.08-1.56)$ & $<0.01$ & & & $1.46(1.16-1.82)$ & $<0.01$ & & \\
\hline BMI & & & $1.28(1.06-1.54)$ & 0.01 & & & $1.44(1.16-1.81)$ & $<0.01$ \\
\hline BMI & & & $1.15(0.93-1.41)$ & 0.19 & & & $1.20(0.94-1.53)$ & 0.14 \\
\hline \multicolumn{9}{|c|}{ Hypertriglyceridemia } \\
\hline Log ALT & $1.22(1.01-1.47)$ & 0.04 & $1.30(1.07-1.57)$ & $<0.01$ & $1.52(1.17-1.98)$ & $<0.01$ & $1.75(1.34-2.28)$ & $<0.01$ \\
\hline Visceral AT & $1.67(1.37-2.05)$ & $<0.01$ & & & $1.83(1.38-2.41)$ & $<0.01$ & & \\
\hline BMI & & & $1.38(1.14-1.67)$ & $<0.01$ & & & $1.19(0.91-1.55)$ & 0.20 \\
\hline \multicolumn{9}{|c|}{ Low HDL-cholesterol } \\
\hline Log ALT & $1.01(0.73-1.40)$ & 0.96 & $1.14(0.82-1.58)$ & 0.44 & $1.06(0.80-1.40)$ & 0.69 & $1.19(0.91-1.55)$ & 0.20 \\
\hline Visceral AT & $1.58(1.14-2.19)$ & $<0.01$ & & & $2.24(1.68-3.00)$ & $<0.01$ & & \\
\hline BMI & & & $1.10(0.80-1.50)$ & 0.56 & & & $1.57(1.21-2.06)$ & $<0.01$ \\
\hline
\end{tabular}

Data presented the change in odds of having a risk factor per 1-SD increase in Log ALT, Visceral AT, or BMI. All models are adjusted for age, alcohole intake, smoking, taking medications, and menopause (women only). The values of 1-SD are 0.51 and 0.45 for Log ALT, $50 \mathrm{~cm}^{2}$ and $38 \mathrm{~cm}^{2}$ for Visceral AT, and $2.9 \mathrm{~kg} / \mathrm{m}^{2}$ and $3.1 \mathrm{~kg} / \mathrm{m}^{2}$ for BMI in men and women, respectively.

published multiethnic study ${ }^{22)}$. Furthermore, in the group comparisons in this study, men in the low-ALT/ high-visceral AT group, but not the high-ALT/lowvisceral AT group, had a significantly higher odds ratio for hypertriglyceridemia than the reference group (low-ALT/low-visceral AT), suggesting a closer association between visceral AT and hypertriglyceridemia. Previous studies, including ours, have demonstrated a closer link with visceral AT among lipid parameters than other metabolic features, such as blood pressure or the level of C-reactive protein ${ }^{1,2,23)}$. Dyslipidemia therefore appears to have a closer link with visceral AT than ALT across ethnicities.

The association between BMI and HDL-cholesterol was not as strong as that for visceral AT to abolish the association with $\log$ ALT. The associations between BMI and the triglyceride and 2-hour plasma glucose levels also appeared weaker than that with visceral AT, as assessed according to the t-statistic values in the regression analysis. On the other hand, the asso- ciation between BMI and blood pressure appeared stronger than that for visceral AT. Previous studies, including ours, have reported that both subcutaneous AT and visceral AT exhibit independent associations with blood pressure ${ }^{1,24}$. As BMI reflects the combined amount of subcutaneous AT and visceral AT, more so than either of the two AT values alone ${ }^{16)}$, it is natural that BMI is associated with blood pressure more strongly than visceral AT alone. Taken together, the visceral AT and ALT values indeed provide significant information beyond BMI for most metabolic factors, except hypertension.

An elevated ALT level was found to be significantly associated with IGT without a concomitant increase in visceral AT among women in the group comparisons in this study. Kantartzis et al. also reported that liver, but not visceral, fat was found to be an independent determinant of the 2-hour plasma glucose level in a cross-sectional study of 330 white individuals ${ }^{25)}$. Recently, we found that an elevated ALT 
Table 5. Adjusted* odds ratios for having metabolic risk factors among 4 subgroups according to ALT and visceral AT levels

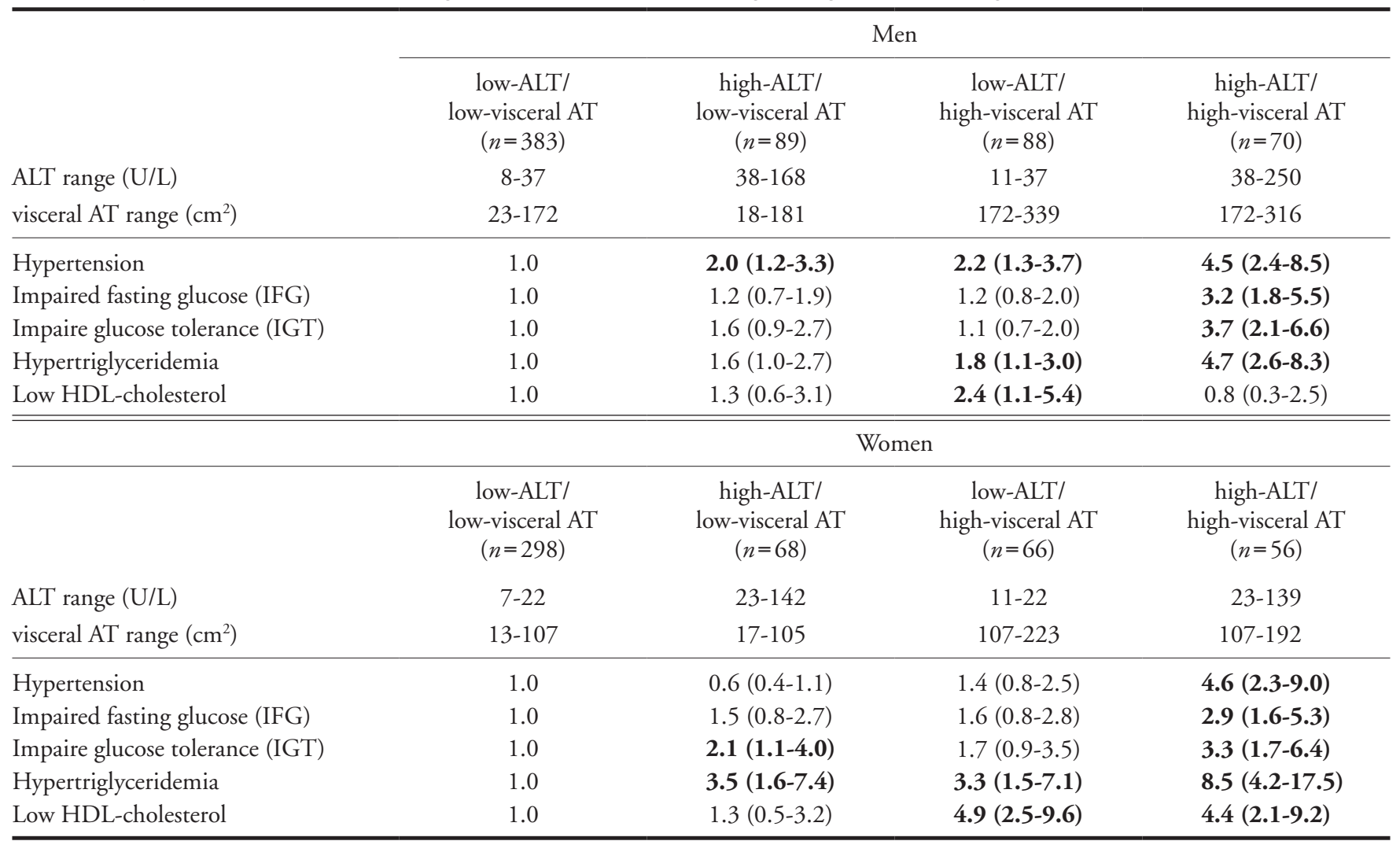

*Adjusted for age, alcohole intake, smoking, taking medications, and menopause (women only). Significantly higher odds ratios compared to the low-ALT/low-visceral AT group are shown in bold letters $(p<0.05)$.

level was independently associated with subsequent progression to IGT among Japanese men with NGT in a longitudinal study ${ }^{26}$. One possible explanation for the association between an elevated ALT level and IGT without increased visceral AT is that increased free fatty acids derived from the lipolysis of intrahepatic triglycerides and/or the increased uptake of plasma free fatty acids in the liver ${ }^{4)}$ can impair the postprandial suppression of hepatic glucose output ${ }^{27}$. Why this closer association was observed only in women in this study remains unknown; however, the relatively smaller amount of visceral AT and lower BMI values noted in women may be involved.

Insulin resistance in the liver may mediate the association between an elevated ALT level and the development of metabolic abnormalities. However, prior studies have reported that adjusting for insulin resistance does not diminish the association between the ALT level and hyperglycemia ${ }^{13}{ }^{26}$ ) or between the ALT level and dyslipidemia ${ }^{28)}$, implying that the ALT level is related to metabolic abnormalities via a different route than insulin resistance. A recent study found that the serum dipeptidyl peptidase-4 (DPP-4) activity is increased, with a positive correlation with the ALT level, in patients with NAFLD ${ }^{29)}$. Dysfunction of the entero-insular axis is one possible explanation for the association between an elevated ALT level and glucose intolerance.

Ethnic differences in body fat distribution have been noted. Asians are characterized by a higher amount of liver ${ }^{15)}$ and visceral fat ${ }^{30,31)}$ than Caucasians, despite having lower BMI values. Meanwhile, African-Americans have the lowest levels of liver and visceral fat for any given amount of total body fat ${ }^{32)}$. Although the pattern of fat distribution is far different between Asians and African-Americans, the associations between respective fat indicators and metabolic risk factors agreed well between our study findings and previously reported results for African-Americans ${ }^{19)}$. Indeed, the investigators of the INSPIRE ME IAA trial reported that the presence of visceral AT and liver fat exhibited similar effects on key metabolic features, except for the C-reactive protein levels, among five ethnic groups ${ }^{22)}$. The patterns of associations 
between specific fat depots and individual risk factors are likely to be shared across ethnicities.

Several limitations associated with this study should be considered. First, the participants were recruited from a population engaged in a specific profession, not from a community-based sample, which may have resulted in selection bias. The role of the "healthy worker effect" should also be considered when generalizing these results to other populations. Second, the cross-sectional design could not be used to examine the causal or temporal sequence between the ALT level and the development of metabolic risk factors. Third, the liver fat content was assessed according to the serum ALT level, not the findings of liver biopsies or imaging examinations. However, there was a low possibility of elevated ALT levels due to alcoholic liver disease or virus hepatitis because the results remained essentially unchanged when the subjects were confined to non-drinkers (Supplementary Table), and subjects positive for viral markers were excluded from this study. ALT is the liver enzyme most closely related to the liver fat content, as measured using proton spectroscopy ${ }^{10,33)}$. Moreover, it is considered to be a readily available biomarker of shifts in fat distribution to ectopic stores, including those in the liver ${ }^{34)}$. Finally, as we conducted several regression analyses, the possibility cannot be excluded that one or more of our results reached a level of statistical significance by chance.

In conclusion, the ALT level was found to exhibit independent associations with most metabolic risk factors after adjusting for visceral AT. In addition, the amount of visceral AT demonstrated a dominant association with HDL-cholesterol in both genders, and the ALT level appeared to have a closer association with IGT in women in this population.

\section{Acknowledgements}

We thank the participants for their cooperation in this study, as well as the staff at the Health Check Department of Hokuriku Central Hospital.

\section{Funding}

The study was supported by the Japan Health Promotion Foundation and a Grant-in-Aid from the Japan Medical Women's Association.

\section{Conflicts of Interest}

None.

\section{References}

1) Fox CS, Massaro JM, Hoffmann U, Pou KM, MaurovichHorvat P, Liu CY, Vasan RS, Murabito JM, Meigs JB, Cupples LA, D’Agostino RB Sr, O’Donnell CJ: Abdominal visceral and subcutaneous adipose tissue compartments: association with metabolic risk factors in the Framingham Heart Study. Circulation, 2007; 116: 39-48

2) Oka R, Kobayashi J, Inazu A, Yagi K, Miyamoto S, Sakurai M, Nakamura K, Miura K, Nakagawa H, Yamagishi $\mathrm{M}$ : Contribution of visceral adiposity and insulin resistance to metabolic risk factors in Japanese men. Metabolism, 2010; 59: 748-754

3) Kotronen A, Yki-Jarvinen H: Fatty liver: a novel component of the metabolic syndrome. Arterioscler Thromb Vasc Biol, 2008; 28: 27-38

4) Fabbrini E, Magkos F, Mohammed BS, Pietka T, Abumrad NA, Patterson BW, Okunade A, Klein S: Intrahepatic fat, not visceral fat, is linked with metabolic complications of obesity. Proc Natl Acad Sci U S A, 2009; 106: 15430-15435

5) Postic C, Dentin R, Girard J: Role of the liver in the control of carbohydrate and lipid homeostasis. Diabetes Metab, 2004; 30: 398-408

6) Hwang JH, Stein DT, Barzilai N, Cui MH, Tonelli J, Kishore P, Hawkins M: Increased intrahepatic triglyceride is associated with peripheral insulin resistance: in vivo MR imaging and spectroscopy studies. Am J Physiol Endocrinol Metab, 2007; 293: E1663-1669

7) Korenblat KM, Fabbrini E, Mohammed BS, Klein S: Liver, muscle, and adipose tissue insulin action is directly related to intrahepatic triglyceride content in obese subjects. Gastroenterology, 2008; 134: 1369-1375

8) Adiels M, Taskinen MR, Packard C, Caslake MJ, SoroPaavonen A, Westerbacka J, Vehkavaara S, Hakkinen A, Olofsson SO, Yki-Jarvinen H, Boren J: Overproduction of large VLDL particles is driven by increased liver fat content in man. Diabetologia, 2006; 49: 755-765

9) Chang Y, Ryu S, Sung E, Jang Y: Higher concentrations of alanine aminotransferase within the reference interval predict nonalcoholic fatty liver disease. Clin Chem, 2007; 53: 686-692

10) Kotronen A, Yki-Jarvinen H, Sevastianova K, Bergholm R, Hakkarainen A, Pietilainen KH, Juurinen L, Lundbom $\mathrm{N}$, Sorensen TI: Comparison of the relative contributions of intra-abdominal and liver fat to components of the metabolic syndrome. Obesity (Silver Spring), 2011; 19: 23-28

11) Hanley AJ, Williams K, Festa A, Wagenknecht LE, D'Agostino RB Jr, Haffner SM: Liver markers and development of the metabolic syndrome: the insulin resistance atherosclerosis study. Diabetes, 2005; 54: 3140-3147

12) Schindhelm RK, Dekker JM, Nijpels G, Stehouwer CD, Bouter LM, Heine RJ, Diamant M: Alanine aminotransferase and the 6-year risk of the metabolic syndrome in Caucasian men and women: the Hoorn Study. Diabet Med, 2007; 24: 430-435

13) Vozarova B, Stefan N, Lindsay RS, Saremi A, Pratley RE, Bogardus C, Tataranni PA: High alanine aminotransferase is associated with decreased hepatic insulin sensitivity and 
predicts the development of type 2 diabetes. Diabetes, 2002; 51: 1889-1895

14) Porter SA, Pedley A, Massaro JM, Vasan RS, Hoffmann U, Fox CS: Aminotransferase levels are associated with cardiometabolic risk above and beyond visceral fat and insulin resistance: the framingham heart study. Arterioscler Thromb Vasc Biol, 2013; 33: 139-146

15) Azuma K, Kadowaki T, Cetinel C, Kadota A, El-Saed A, Kadowaki S, Edmundowicz D, Nishio Y, Sutton-Tyrrell K, Okamura T, Evans RW, Takamiya T, Ueshima H, Curb JD, Abbott RD, Kuller LH, Kelley DE, Sekikawa A: Higher liver fat content among Japanese in Japan compared with non-Hispanic whites in the United States. Metabolism, 2009; 58: 1200-1207

16) Oka R, Miura K, Sakurai M, Nakamura K, Yagi K, Miyamoto S, Moriuchi T, Mabuchi H, Yamagishi M, Takeda Y, Hifumi S, Inazu A, Nohara A, Kawashiri MA, Kobayashi $\mathrm{J}$ : Comparison of waist circumference with body mass index for predicting abdominal adipose tissue. Diabetes Res Clin Pract, 2009; 83: 100-105

17) Grundy SM, Cleeman JI, Daniels SR, Donato KA, Eckel RH, Franklin BA, Gordon DJ, Krauss RM, Savage PJ, Smith SC Jr, Spertus JA, Costa F: Diagnosis and management of the metabolic syndrome: an American Heart Association/National Heart, Lung, and Blood Institute Scientific Statement. Circulation, 2005; 112: 2735-2752

18) Kobayashi J, Tadokoro N, Watanabe M, Shinomiya M: A novel method of measuring intra-abdominal fat volume using helical computed tomography. Int J Obes Relat Metab Disord, 2002; 26: 398-402

19) Liu J, Fox CS, Hickson D, Bidulescu A, Carr JJ, Taylor HA: Fatty liver, abdominal visceral fat, and cardiometabolic risk factors: the Jackson Heart Study. Arterioscler Thromb Vasc Biol, 2011; 31: 2715-2722

20) Kim HJ, Kim HJ, Lee KE, Kim DJ, Kim SK, Ahn CW, Lim SK, Kim KR, Lee HC, Huh KB, Cha BS: Metabolic significance of nonalcoholic fatty liver disease in nonobese, nondiabetic adults. Arch Intern Med, 2004; 164: 2169-2175

21) Magkos F, Fabbrini E, Mohammed BS, Patterson BW, Klein S: Increased whole-body adiposity without a concomitant increase in liver fat is not associated with augmented metabolic dysfunction. Obesity (Silver Spring), 2010; 18: 1510-1515

22) Nazare JA, Smith JD, Borel AL, Haffner SM, Balkau B, Ross R, Massien C, Almeras N, Despres JP: Ethnic influences on the relations between abdominal subcutaneous and visceral adiposity, liver fat, and cardiometabolic risk profile: the International Study of Prediction of IntraAbdominal Adiposity and Its Relationship With Cardiometabolic Risk/Intra-Abdominal Adiposity. Am J Clin Nutr, 2012; 96: 714-726

23) Grundy SM, Adams-Huet B, Vega GL: Variable contributions of fat content and distribution to metabolic syndrome risk factors. Metab Syndr Relat Disord, 2008; 6:
281-288

24) Oka R, Miura K, Sakurai M, Nakamura K, Yagi K, Miyamoto S, Moriuchi T, Mabuchi H, Koizumi J, Nomura H, Takeda Y, Inazu A, Nohara A, Kawashiri MA, Nagasawa S, Kobayashi J, Yamagishi M: Impacts of visceral adipose tissue and subcutaneous adipose tissue on metabolic risk factors in middle-aged Japanese. Obesity (Silver Spring), 2010; 18: 153-160

25) Kantartzis K, Machann J, Schick F, Fritsche A, Haring HU, Stefan N: The impact of liver fat vs visceral fat in determining categories of prediabetes. Diabetologia, 2010; 53: 882-889

26) Oka R, Aizawa T, Yagi K, Hayashi K, Kawashiri M, Yamagishi $\mathrm{M}$ : Elevated liver enzymes are related to progression to impaired glucose tolerance in Japanese men. Diabet Med, 2013;

27) Singhal P, Caumo A, Carey PE, Cobelli C, Taylor R: Regulation of endogenous glucose production after a mixed meal in type 2 diabetes. Am J Physiol Endocrinol Metab, 2002; 283: E275-283

28) Lorenzo C, Hanley AJ, Rewers MJ, Haffner SM: The association of alanine aminotransferase within the normal and mildly elevated range with lipoproteins and apolipoproteins: the Insulin Resistance Atherosclerosis Study. Diabetologia, 2013; 56: 746-757

29) Firneisz G, Varga T, Lengyel G, Feher J, Ghyczy D, Wichmann B, Selmeci L, Tulassay Z, Racz K, Somogyi A: Serum dipeptidyl peptidase- 4 activity in insulin resistant patients with non-alcoholic fatty liver disease: a novel liver disease biomarker. PLoS One, 2010; 5: e12226

30) Lear SA, Humphries KH, Kohli S, Chockalingam A, Frohlich JJ, Birmingham CL: Visceral adipose tissue accumulation differs according to ethnic background: results of the Multicultural Community Health Assessment Trial (M-CHAT). Am J Clin Nutr, 2007; 86: 353-359

31) Kadowaki T, Sekikawa A, Murata K, Maegawa H, Takamiya T, Okamura T, El-Saed A, Miyamatsu N, Edmundowicz D, Kita Y, Sutton-Tyrrell K, Kuller LH, Ueshima $\mathrm{H}$ : Japanese men have larger areas of visceral adipose tissue than Caucasian men in the same levels of waist circumference in a population-based study. Int $\mathrm{J}$ Obes (Lond), 2006; 30: 1163-1165

32) Guerrero R, Vega GL, Grundy SM, Browning JD: Ethnic differences in hepatic steatosis: an insulin resistance paradox? Hepatology, 2009; 49: 791-801

33) Westerbacka J, Corner A, Tiikkainen M, Tamminen M, Vehkavaara S, Hakkinen AM, Fredriksson J, Yki-Jarvinen $\mathrm{H}$ : Women and men have similar amounts of liver and intra-abdominal fat, despite more subcutaneous fat in women: implications for sex differences in markers of cardiovascular risk. Diabetologia, 2004; 47: 1360-1369

34) Ioannou GN: Implications of elevated serum alanine aminotransferase levels: think outside the liver. Gastroenterology, 2008; 135: 1851-1854 


\section{Supplementary Table}

Multivariable-adjusted* regressions for continuous metabolic risk factors with $\log$ ALT andvisceral AT confined to non-drinkers

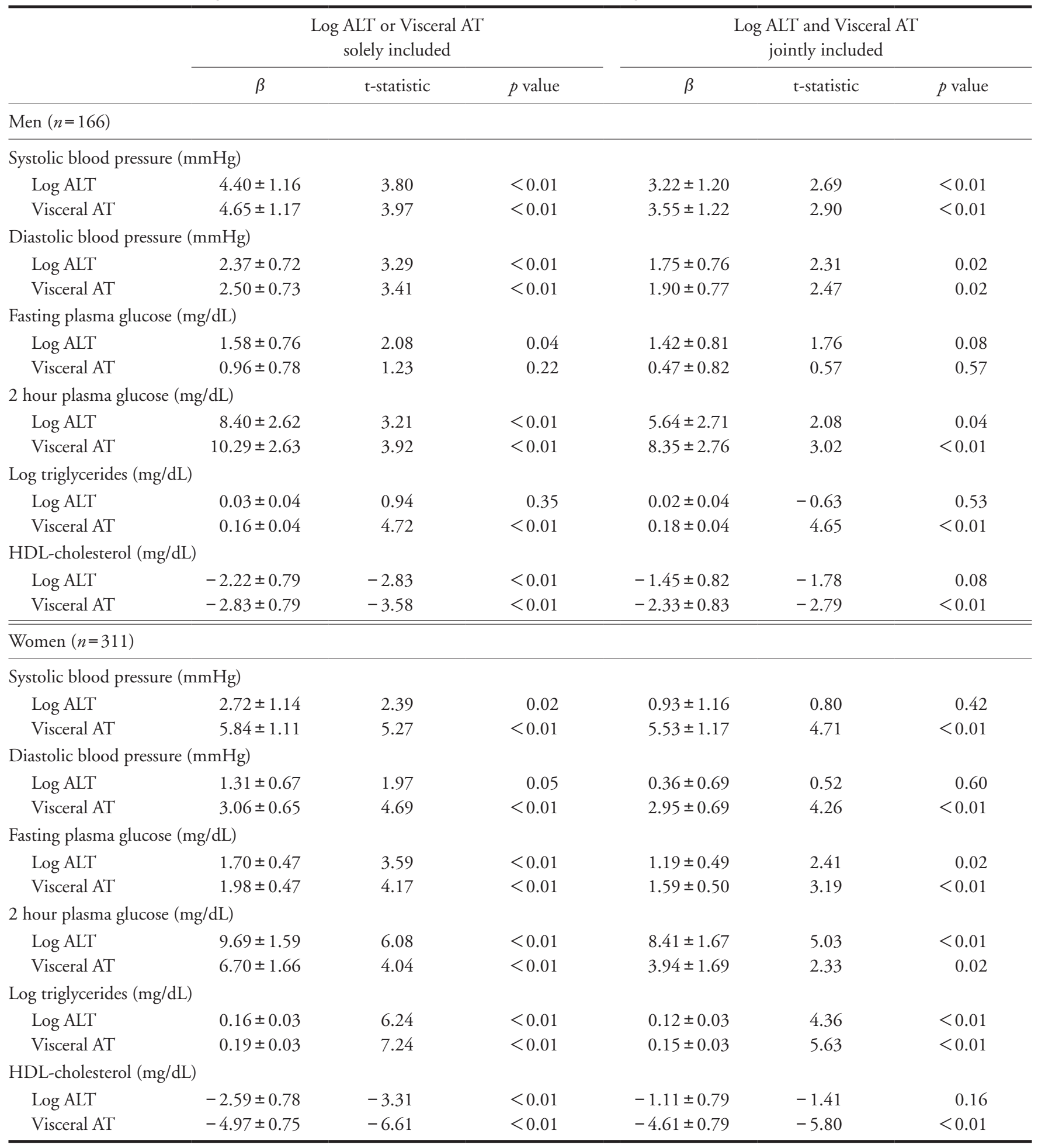

*All regression models are adjusted for age, alcohole intake, smoking, taking medications, and menopause (women only). $\beta$ denotes the average change $( \pm \mathrm{SE})$ in risk factor per 1-SD increase in Log ALT or Visceral AT. The values of 1-SD are 0.52 and 0.47 for Log ALT and $52 \mathrm{~cm}^{2}$ and 38 $\mathrm{cm}^{2}$ for Visceral AT in men and women, respectively. 